\title{
PERMEÂMETRO DE CARGA DECRESCENTE ASSOCIADO A PROGRAMA COMPUTACIONAL PARA A DETERMINAÇÃO DA CONDUTIVIDADE HIDRÁULICA DO SOLO SATURADO ${ }^{(1)}$
}

\author{
Paulo Ivonir Gubiani ${ }^{(2)}$, Dalvan José Reinert ${ }^{(3)}$, José Miguel Reichert ${ }^{(3)}$, Neiva \\ Somavilla Gelain ${ }^{(4)}$ \& Jean Paolo Gomes Minella ${ }^{(3)}$
}

\begin{abstract}
RESUMO
A condutividade hidráulica do solo saturado $\left(\mathrm{K}_{\theta \mathrm{s}}\right)$ é uma propriedade com grande variabilidade, o que exige a utilização de um número maior de determinações para que sua descrição possa ser feita adequadamente pela função densidade de probabilidade normal. Consequentemente, há aumento de trabalho e de tempo para a obtenção dos resultados, principalmente se as determinações forem feitas com equipamentos de pouca praticidade. A construção de equipamentos de maior praticidade e o desenvolvimento de ferramentas computacionais podem tornar o processo de análise mais rápido e preciso. Com esse objetivo, foi construído um permeâmetro de carga decrescente e desenvolvido um software para a aquisição de dados. As medidas de $K_{\theta \mathrm{s}}$ obtidas com esses equipamentos, em amostras de um Argissolo, mostraram menor variabilidade, avaliada pelo coeficiente de variação, o que resultou em maior precisão das determinações. Além disso, o tempo de análise foi reduzido em $30 \%$.
\end{abstract}

Termos de indexação: propriedades hídricas, software, métodos.
SUMMARY: FALLING HEAD PERMEAMETER AND SOFTWARE TO DETERMINE THE HYDRAULIC CONDUCTIVITY OF SATURATED SOIL

The soil saturated hydraulic conductivity $\left(K_{\theta s}\right)$ is a property with great variability, which requires the use of a greater number of determinations so that they can be described by the normal probability density function. Consequently, there is an increase in time and labor to

\footnotetext{
(1) Recebido para publicação em abril de 2009 e aprovado em março de 2010.

(2) Doutorando do Curso de Pós-Graduação em Ciência do Solo (PPGCS), Universidade Federal de Santa Maria - UFSM. Campus Universitário, CEP 97105-900 Santa Maria (RS). Bolsista CAPES. E-mail: paulogubiani@gmail.com

(3) Professor, Departamento de Solos, CCR, Universidade Federal de Santa Maria - UFSM. Av. Roraima 1000, CEP 97105-900 Santa Maria (RS).Pesquisador do CNPq. E-mail: dalvan@ccr.ufsm.br; reichert@smail.ufsm.br; jminella@smail.ufsm.br

(4) Graduanda do Curso de Engenharia Florestal, UFSM. Bolsista FAPERGS. E-mail: neivaengenheira@gmail.com
} 


\begin{abstract}
obtain $K_{\theta s}$ results if determined by conventional equipment. The use of more practical equipment and computational tools allows a faster and more accurate analysis. With this aim a falling head permeameter was built and a software for data acquisition was developed. Values of $K_{\theta s}$ obtained with this equipment in Hapludalf samples showed less variability, as assessed by the coefficient of variation, resulting in more precise measurements. Moreover, the time of analysis was reduced by $30 \%$.
\end{abstract}

Index terms: hydric properties, software, methods.

A condutividade hidráulica de solo saturado $\left(\mathrm{K}_{\theta \mathrm{s}}\right)$ é uma medida de grande importância, pois indica a qualidade estrutural do solo, pelo fato de estar diretamente relacionada com a geometria e a continuidade dos poros preenchidos com água (Mesquita \& Moraes, 2004). Além disso, a $K_{\theta \mathrm{s}}$ é considerada um dos parâmetros físico-hídricos mais importantes de modelos utilizados no dimensionamento de sistemas de drenagem (Oliveira et al., 2003).

A descrição da $K_{\theta s}$ pela função densidade de probabilidade normal, ou funções aproximadas a esta, exige um número maior de amostras, devido à sua grande variabilidade (Mesquita \& Moraes, 2003). Conforme Eguchi et al. (2003), se essa variação não for corretamente observada, o planejamento do manejo do solo, a eficiência de projetos de irrigação e drenagem e os resultados de pesquisas podem ser comprometidos.

$\mathrm{O}$ aumento do número de amostras tem algumas implicações, sobretudo de ordem prática. Os métodos correntes disponíveis para a medição da $\mathrm{K}_{\theta \mathrm{s}}$ não são facilmente usados repetidamente, pelo alto custo, por consumirem tempo ou serem complicados tecnicamente (Mesquita \& Moraes, 2004). Novas estratégias para a medição da $\mathrm{K}_{\theta \mathrm{s}}$ devem, portanto, atender a requisitos como reduzir o trabalho, possuir mecanismos para organizar e processar maior quantidade de informações e diminuir as chances de erro. Essas características podem ser obtidas com a inclusão de ferramentas computacionais nos permeâmetros, o que tornaria o processo de análise mais dinâmico, prático e seguro.

Os objetivos deste trabalho foram descrever a montagem e utilização de um permeâmetro de carga decrescente associado a programa computacional e testar a precisão e a praticidade na determinação da condutividade hidráulica de solo saturado.

O solo utilizado para os testes é classificado como Argissolo Vermelho distrófico típico (Embrapa, 2006). As amostras foram coletadas com anéis metálicos (5,72 cm de diâmetro e 4,0 cm de altura) na camada de $0-10 \mathrm{~cm}$, saturadas e divididas em três grupos para a medida da $\mathrm{K}_{\theta \mathrm{s}}$, com diferentes estratégias: (a) permeâmetro de carga decrescente associado ao programa computacional (12 amostras); (b) permeâmetro de carga decrescente, com tomada de tempo por meio de cronômetro manual (12 amostras); e (c) permeâmetro de carga constante (24 amostras).
O permeâmetro de carga decrescente foi construído com base em um modelo alemão desenvolvido no Instituto de Nutrição de Plantas e Ciência do Solo da Christian Albrechts Universität zu Kiel (Hartge \& Horn, 1998). O equipamento é composto de duas partes principais (Figura 1). Uma das partes é constituída pela cúpula metálica ligada a dutos de alimentação de ar e água. $\mathrm{O}$ anel metálico, que contém a amostra de solo saturado, tem dimensões de 7,5 cm de diâmetro e $4 \mathrm{~cm}$ de altura, sendo fixado no interior da cúpula por meio de uma borracha inflável de vedação. $\mathrm{O}$ ar comprimido proveniente do compressor passa por um manômetro, que regula a pressão aplicada na borracha para valores em torno de $50 \mathrm{kPa}$. Além de fixar a amostra de solo na cúpula, a vedação tem por função impedir a passagem de água entre a parte externa do anel contendo a amostra de solo e as laterais da cúpula. Um dispositivo localizado na parte superior e que se comunica com o interior da cúpula permite a retirada de ar, que pode ficar aprisionado no espaço entre o topo da cúpula e a superfície da amostra de solo.

A outra parte consiste de três tubos de vidro graduados ( $\mathrm{P}, \mathrm{M}$ e $\mathrm{G})$, dispostos verticalmente $\mathrm{e}$ posicionados de forma que a parte inferior dos tubos coincida aproximadamente com o nível inferior da cúpula. Os tubos são conectados a uma tubulação de PVC flexível, que, de um lado, fornece água para o abastecimento dos tubos e estabelecimento da carga hidráulica e, de outro, conduz a água para a amostra de solo contida na cúpula. A entrada e saída da água dos tubos é controlada por registros acionados manualmente. Os diâmetros internos dos tubos são de $3,95 \mathrm{~mm}(\mathrm{P}), 5,91 \mathrm{~mm}(\mathrm{M})$ e $10,02 \mathrm{~mm}(\mathrm{G})$ e permitem, do maior para o menor diâmetro, diminuir o volume de água que passa pela amostra sem modificar a carga hidráulica. Com isso, o tempo de fluxo de água na amostra é reduzido - estratégia importante para amostras com $\mathrm{K}_{\theta \mathrm{s}}$ baixa.

Em operação, a cúpula contendo a amostra de solo é colocada submersa no interior de um recipiente sobre uma malha metálica, para evitar o contato direto da amostra de solo com o fundo do recipiente, o que dificultaria a saída de água que flui pela amostra. A carga hidráulica inicial ou no limite superior $\left(\mathrm{H}_{0}=54,7 \mathrm{~cm}\right)$ é estabelecida pela diferença entre $\mathrm{o}$ nível da água nos tubos e o nível da água no recipiente. A carga hidráulica final $\left(\mathrm{H}_{3}=24,7 \mathrm{~cm}\right)$ é definida $30 \mathrm{~cm}$ abaixo do limite superior. Esses limites podem 


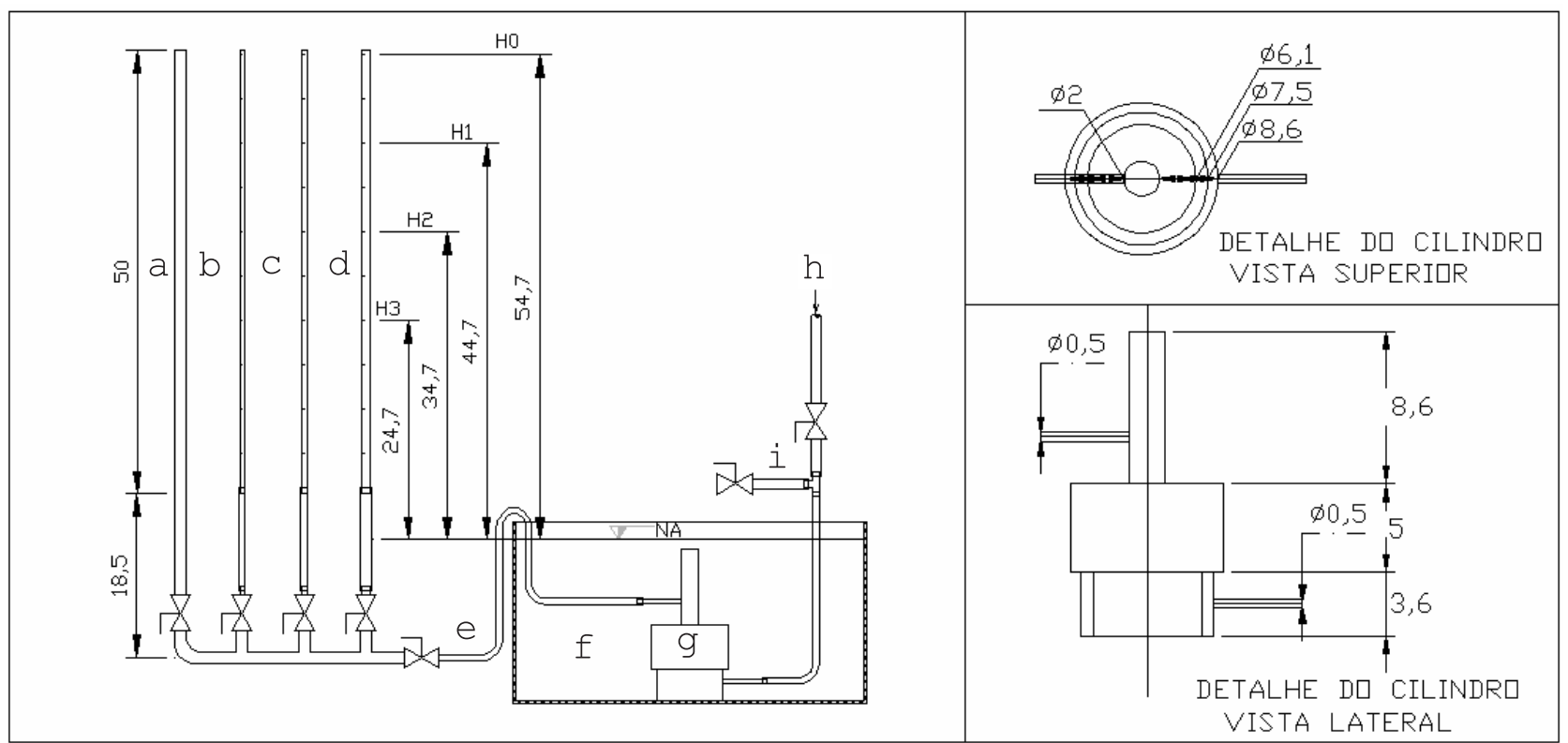

Figura 1. Permeâmetro de carga decrescente. Tubulação de alimentação de água (a); tubos de vidro graduados, com diâmetro interno de $3,95 \mathrm{~mm}$ (b), 5,91 $\mathrm{mm}$ (c) e 10,02 $\mathrm{mm}$ (d); segmento do duto de PVC flexível que alimenta a cúpula com água (e); tanque preenchido com água (f); cilindro ou cúpula onde é inserida a amostra de solo (g); segmento do duto de PVC flexível que conduz ar pressurizado à borracha de vedação do interior da cúpula (h); duto de despressurização (i). Mais ilustrações e detalhes da montagem do permeâmetro estão disponíveis na página http://www.ufsm.br/fisicadosolo.

ser deslocados para cima (aumento da carga hidráulica), se a amostra conduz pouca água, ou para baixo (diminuição da carga hidráulica), se a amostra for bem permeável. Pelas mesmas razões, o intervalo de $30 \mathrm{~cm}$ pode ser reduzido ou ampliado.

O procedimento de determinação da $K_{\theta s}$ consiste em medir o tempo gasto para a passagem pela amostra de solo do volume de água contido entre os limites superior e inferior do tubo, definidos conforme o intervalo de carga hidráulica considerada. $\mathrm{A} \mathrm{K}_{\theta \mathrm{s}}$ é calculada conforme a equação (Hillel, 1998):

$$
K_{\theta s}=(2,3 a L / A \Delta t)\left(\log H_{n}-\log H_{n+1}\right)
$$

em que: $K_{\theta s}$ é a condutividade hidráulica de solo saturado $\left(\mathrm{cm} \mathrm{h}^{-1}\right) ; H_{n}$ e $H_{n+1}$ são, respectivamente, a carga hidráulica inicial e final $(\mathrm{cm}) ; \Delta t$ é o intervalo de tempo para o decréscimo da carga hidráulica de $H_{n}$ até $H_{n+1}(\mathrm{~h}) ; A$ é a área da seção transversal da amostra de solo $\left(\mathrm{cm}^{2}\right) ; L$ é o comprimento da amostra de solo (cm); e $a$ é a área da seção transversal interna do tubo que contém a coluna de água $\left(\mathrm{cm}^{2}\right)$.

$\mathrm{O}$ aplicativo computacional, denominado de Ksat, foi desenvolvido em Visual Basic 6.0, com banco de dados Acces (Figura 2). O programa foi projetado conforme as características operacionais do permeâmetro de carga decrescente construído no Laboratório de Física do Solo da Universidade Federal de Santa Maria (UFSM) (Figura 1). Entretanto, as configurações do programa podem ser alteradas, o que possibilita a adequação do Ksat a situações distintas, tanto de procedimentos de análises quanto de construção do permeâmetro. O programa facilita a obtenção de mais de uma medida da $\mathrm{K}_{\theta \mathrm{s}}$ para cada descarga da água contida entre o limite superior e o inferior da carga hidráulica predefinida. Isso é feito pela divisão da coluna de água em intervalos de carga hidráulica correspondentes ao número de medidas da $\mathrm{K}_{\theta \mathrm{S}}$ desejadas em cada teste. Essa estratégia possibilita identificar, pela diferença entre os valores de $\mathrm{K}_{\theta \mathrm{s}}$ dos diferentes intervalos, problemas como vazamentos ou alterações da amostra durante o teste. Contudo, o usuário pode configurar o programa para fazer apenas uma medida da $\mathrm{K}_{\theta \mathrm{s}}$ em cada análise.

O processo de aquisição de dados com o Ksat não é automatizado, mas por comandos de teclado. O Ksat atua na tomada dos tempos de decréscimo da carga hidráulica. Neste trabalho, os tempos foram registrados em quatro cargas hidráulicas $\left(\mathrm{H}_{0}, \mathrm{H}_{1}, \mathrm{H}_{2}\right.$ e $\mathrm{H}_{3}$ ), cujos valores são, respectivamente, 54,7; 44,7; 34,7 ; e $24,7 \mathrm{~cm}$, em relação ao nível da água no reservatório (Figuras 1 e 2). Os tempos $\mathrm{T}_{0}, \mathrm{~T}_{1}, \mathrm{~T}_{2} \mathrm{e}$ $\mathrm{T}_{3}$ foram registrados por quatro pressionamentos da tecla "Enter", em $\mathrm{H}_{0}, \mathrm{H}_{1}, \mathrm{H}_{2}$ e $\mathrm{H}_{3}$. Dessa forma, foram calculados três valores de $\mathrm{K}_{\theta \mathrm{s}}$ para cada amostra (entre $\mathrm{H}_{0}-\mathrm{H}_{1}, \mathrm{H}_{1}-\mathrm{H}_{2}$ e $\mathrm{H}_{2}-\mathrm{H}_{3}$ ) sem interromper o decréscimo da carga hidráulica, cujos resultados foram usados para calcular o desvio-padrão das medidas. Como o permeâmetro de carga decrescente permite processar uma única amostra por vez, o tempo total gasto nas 


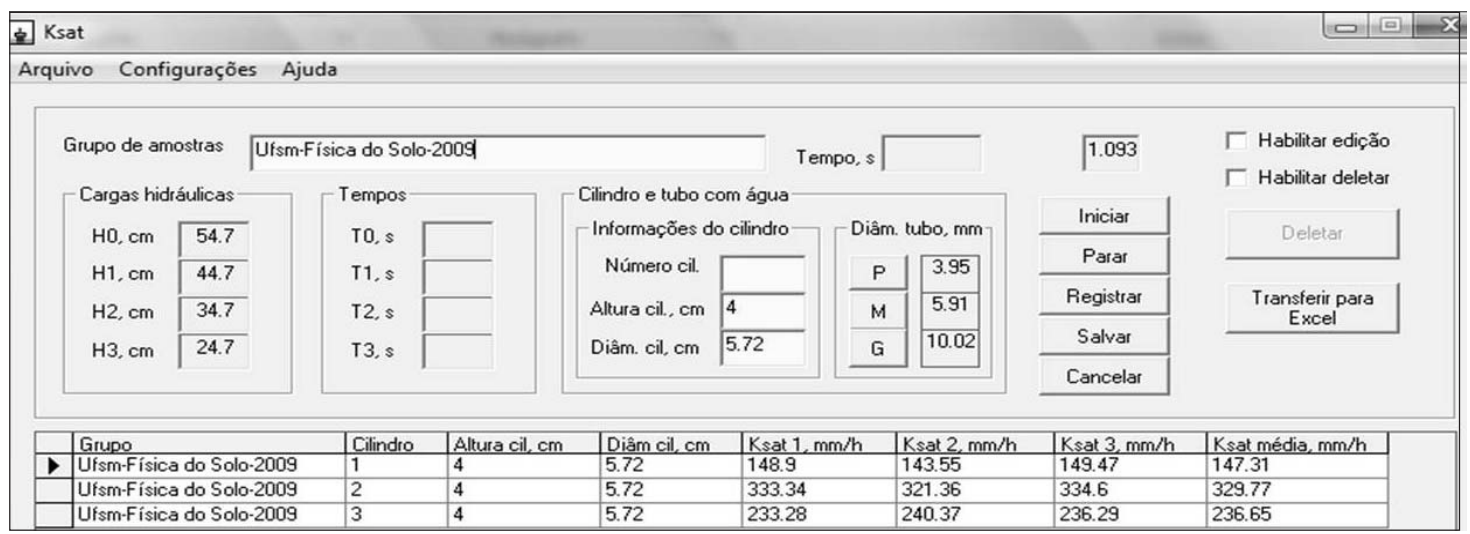

Figura 2. Tela principal do Ksat.

medições foi o somatório do tempo de cada amostra individual.

Nas determinações feitas com o permeâmetro de carga decrescente e registro do tempo com cronômetro manual, o decréscimo da carga hidráulica foi interrompido duas vezes (logo após as alturas $\mathrm{H}_{1}$ e $\mathrm{H}_{2}$ ) para a tomada das três medidas da $\mathrm{K}_{\theta \mathrm{s}}$ por teste. Isso acontece porque o cronômetro disparado em $\mathrm{H}_{0}$ ou $\mathrm{H}_{1}$ precisa ser parado em $\mathrm{H}_{1}$ ou $\mathrm{H}_{2}$, respectivamente, para registrar o tempo de decréscimo da carga hidráulica entre $\mathrm{H}_{0}-\mathrm{H}_{1} \mathrm{e} \mathrm{H}_{1}-\mathrm{H}_{2}$. Além disso, após o registro do tempo em $\mathrm{H}_{1}$ e $\mathrm{H}_{2}$, a carga hidráulica deve ser retornada a um ponto acima dessas posições, para o registro do tempo inicial do intervalo seguinte. Dessa forma, foram registrados três pares de tempo, que incluem os tempos iniciais e finais dos intervalos $\mathrm{H}_{0}-\mathrm{H}_{1}, \mathrm{H}_{1}-\mathrm{H}_{2}$ e $\mathrm{H}_{2}-\mathrm{H}_{3}$.

No permeâmetro de carga constante (Embrapa, 1997), as três medidas do volume de água percolada em cada amostra previamente saturada foram feitas a cada cinco minutos, após o início da percolação. A estrutura do permeâmetro usado permite processar 12 amostras por vez. Por isso, o tempo total não é igual ao número de amostras multiplicado por $15 \mathrm{~min}$ (cinco minutos por medida), porque o tempo gasto relativo a cada amostra individual é igual ao tempo total gasto dividido pelo número de amostras (tempo por amostra $=$ tempo total $/ 24$ ).

As medidas da $\mathrm{K}_{\theta \mathrm{s}}$ obtidas com as diferentes estratégias foram comparadas por meio do coeficiente de variação (CV), calculado para cada amostra a partir das três medidas da $\mathrm{K}_{\theta \mathrm{s}}$ feitas na mesma amostra. Se medidas sucessivas são feitas na mesma amostra, o método mais preciso é aquele que gera menor variação entre os valores medidos. A precisão dos métodos foi avaliada pela frequência de distribuição do CV nas classes de $0-5,5-10,10-20$ e $20-30 \%$, intervalos esses definidos para permitir melhor visualização da variabilidade de cada método. A praticidade na execução das medidas foi avaliada pelo tempo gasto nas determinações.
Com o uso do permeâmetro de carga decrescente associado ao Ksat, 80 \% dos CVs se distribuíram na classe de $0-5 \%$ (Figura 3 ), e o restante $(20 \%)$ permaneceu na classe de 5-10 \%. Quando o Ksat foi substituído pelo cronômetro manual, para registro dos tempos de decréscimo da carga hidráulica, a frequência dos CVs foi de 84,6, 7,7 e 7,7 \%, respectivamente nas classes $0-5,5-10$ e $10-20 \%$, indicando maior variabilidade dessa estratégia de medida.

A diferença na variabilidade dessas duas estratégias de medida pode ser explicada pelas características do procedimento de registro dos tempos de decréscimo da carga hidráulica. Com o Ksat, o decréscimo da carga hidráulica não foi interrompido para a tomada dos tempos (a tecla "Enter" foi pressionada três vezes). Com o cronômetro manual, o decréscimo da carga hidráulica foi interrompido duas vezes e, por isso, foram feitos seis acionamentos no cronômetro (três inicializações e três paradas). Dessa forma, as chances de erro no registro do tempo foram maiores (por retardamento ou adiantamento dos acionamentos) e se refletiram na variabilidade das medidas.

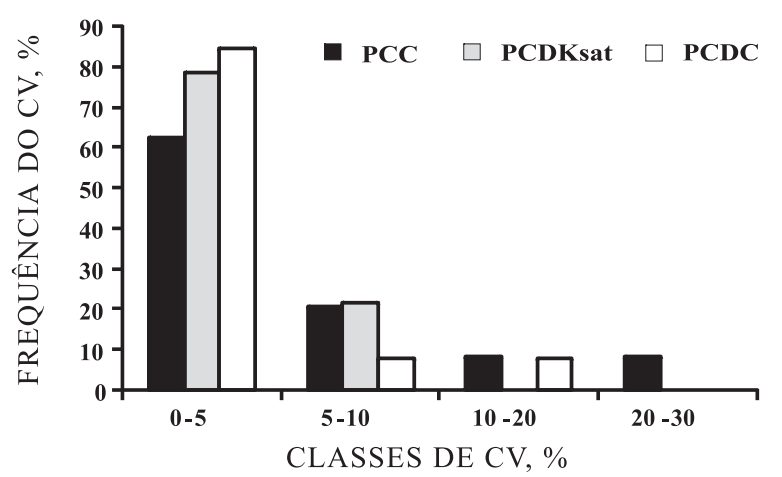

Figura 3. Distribuição do coeficiente de variação (CV) por classes. PCC: permeâmetro de carga constante; PCDKsat: permeâmetro de carga decrescente associado ao Ksat; e PCDC: permeâmetro de carga decrescente e cronômetro manual. 
A maior variabilidade nas medidas de $\mathrm{K}_{\theta \mathrm{s}}$ foi observada com o uso do permeâmetro de carga constante. Apenas $62,5 \%$ do CV ficou compreendido na classe de $0-5 \%$. Como consequência, $47,3 \%$ do CV distribuiu-se em classes maiores, e $8,3 \%$ do CV foi verificado na classe de $20-30 \%$. Isso mostra que houve considerável variação das medidas para uma mesma amostra, o que indica menor precisão do método. Esse resultado deve estar associado às variações do tempo de posicionamento e retirada do recipiente coletor de água, bem como a erros de leitura (feitos em proveta) do volume percolado.

O tempo necessário para a execução de cada teste também foi um fator analisado. Considerando um conjunto de 100 amostras, os tempos para a determinação da condutividade hidráulica foram de 245,380 e $350 \mathrm{~min}$, respectivamente para o permeâmetro de carga decrescente associado ao Ksat, permeâmetro de carga decrescente com cronômetro manual e permeâmetro de carga constante. Assim, em relação ao permeâmetro de carga constante, o uso do permeâmetro de carga decrescente associado ao Ksat reduziu em 30 \% o tempo de análise, enquanto o permeâmetro de carga decrescente com uso de cronômetro manual aumentou esse tempo em $9 \%$. Além de o tempo gasto nas determinações feitas com a segunda e terceira estratégias de medida ter sido maior, uma segunda pessoa foi necessária para fazer as anotações em ficha de papel. Cabe ressaltar que o tempo gasto no permeâmetro de carga constante deveu-se à quantidade de amostras processadas por vez. Permeâmetros com capacidade de processar mais que 12 (caso deste estudo) reduzirão esse tempo por amostra.

Outra grande vantagem do uso do Ksat é a possibilidade de verificar o resultado imediatamente após a análise, o que permite corrigir possíveis erros ou comprovar o valor obtido pela repetição do teste. A obtenção de valores discrepantes (altos) para $\mathrm{K}_{\theta \mathrm{s}}$ é comum, devido à sua característica variável (Mesquita et al., 2003). Se esses valores forem conhecidos somente num momento posterior, e se não houver possibilidade de repetir o teste, eles podem ser excluídos do conjunto. A repetição do teste torna mais segura a decisão de manter ou eliminar a amostra.

$\mathrm{O}$ arquivo "ajuda" do Ksat fornece informações relativamente detalhadas sobre todos os mecanismos e configurações do programa e apresenta um exemplo da sequência a ser seguida, para a realização das análises. O programa está disponível para download na página http://www.ufsm.br/fisicadosolo, onde, também, estão disponíveis ilustrações com detalhes da montagem do permeâmetro.
O uso do permeâmetro de carga decrescente associado ao Ksat reduz o tempo de análise da $\mathrm{K}_{\theta \mathrm{s}} \mathrm{e}$ possibilita a verificação dos resultados em tempo real, o que confere maior praticidade nas determinações. Por ser o método que apresentou a menor variabilidade, conclui-se que essa estratégia é mais precisa na determinação da $\mathrm{K}_{\theta \mathrm{s}}$.

\section{AGRADECIMENTOS}

Ao professor Rainer Horn, do Institute of Plant Nutrition and Soil Science da Christian Albrechts University zu Kiel - Alemanha, pela disponibilização das características técnicas do permeâmetro. Ao Engenheiro Agrícola André Anibal Brandt e ao Engenheiro Agrônomo Douglas Rodrigo Kaiser (UFSM), pela montagem do permeâmetro. À engenheira civil Nadia Bernardi Bonumá (UFSM), pela elaboração dos desenhos.

\section{LITERATURA CITADA}

EGUCHI, E.S.; SILVA, E.L. \& OLIVEIRA, M.S. Variabilidade espacial da condutividade hidráulica do solo saturado e da taxa de infiltração básica determinadas in situ. Ci. Agrotec., 23:1607-1613, 2003.

EMPRESA BRASILEIRA DE PESQUISA AGROPECUÁRIA EMBRAPA/CNPS. Sistema Brasileiro de Classificação de Solos. 2.ed. Brasília: Embrapa, 2006, 306p.

EMPRESA BRASILEIRA DE PESQUISA AGROPECUÁRIA EMBRAPA. Centro Nacional de Pesquisa de Solos. Manual de métodos de análise de solo. 2.ed. Rio de Janeiro, 1997. $212 \mathrm{p}$.

HARTGE, K.H. \& HORN, R. Die physikalische Untersuchung von Böden. Ferdinand Enke Verlag, 3. Stuttgart, Auflage, 1989. $177 \mathrm{p}$.

HILLEL, D. Environmental soil physics. São Diego, Academic Press, 1998.

MESQUITA, M.G.B.F. \& MORAES, S.O. A dependência entre a condutividade hidráulica saturada e atributos físicos do solo. Ci. Rural, 34:963-969, 2004.

MESQUiTA, M.G.B.F.; MORAES, S.O. \& CORRENTE, J.E. Caracterização estatística de variáveis físicas do solo. Acta Sci. Agron., 25:35-44, 2003.

OLIVEIRA, L.F.C.; BONONO, R.; CARVALHO, D.F. \& CORTÊS, F.C. Influência da condutividade hidráulica na altura do lençol freático, espaçamento de drenos e na produtividade do milho. Biosci. J., 19:35-41, 2003. 